\title{
Next-to-leading-order QCD corrections to heavy quark fragmentation into ${ }^{1} S_{0}^{(1,8)}$ quarkonia
}

\author{
Feng Feng ${ }^{1,2,3, a}$, Yu Jia ${ }^{3,4, b}$, Wen-Long Sang ${ }^{1, \mathfrak{c}} \mathbb{C}$ \\ ${ }^{1}$ School of Physical Science and Technology, Southwest University, Chongqing 400700, China \\ ${ }^{2}$ China University of Mining and Technology, Beijing 100083, China \\ ${ }^{3}$ Institute of High Energy Physics, Chinese Academy of Sciences, Beijing 100049, China \\ ${ }^{4}$ School of Physics, University of Chinese Academy of Sciences, Beijing 100049, China
}

Received: 4 June 2021 / Accepted: 25 June 2021 / Published online: 9 July 2021

(C) The Author(s) 2021

\begin{abstract}
Within NRQCD factorization framework, in this work we compute, at the lowest order in velocity expansion, the next-to-leading-order (NLO) perturbative corrections to the short-distance coefficients associated with heavy quark fragmentation into the ${ }^{1} S_{0}^{(1,8)}$ components of a heavy quarkonium. Starting from the Collins and Soper's operator definition of the quark fragmentation function, we apply the sector decomposition method to facilitate the numerical manipulation. It is found that the NLO QCD corrections have a significant impact.
\end{abstract}

The large- $p_{\perp}$ production of an identified hadron $H$ in high-energy collision is dominated by the so-called fragmentation mechanism. According to the celebrated QCD factorization theorem, the inclusive production rate of the $H$ with large $p_{\perp}$ at hadron collider can be cast into the following factorized form [1]:

$$
\begin{aligned}
& d \sigma\left[A+B \rightarrow H\left(P_{\perp}\right)+X\right] \\
& =\sum_{i} d \hat{\sigma}\left[A+B \rightarrow i\left(P_{\perp} / z\right)+X\right] \\
& \otimes D_{i \rightarrow H}(z, \mu)+\mathcal{O}\left(m_{H}^{2} / P_{\perp}^{2}\right),
\end{aligned}
$$

where $A, B$ represent two colliding hadrons, $d \hat{\sigma}$ denotes the partonic cross section, and the sum in (1) is extended over all parton species $(i=q, \bar{q}, g) . D_{i \rightarrow H}(z)$ characterizes the fragmentation function (FF), encoding the probability for the parton $i$ to hadronize into a multi-hadron state that contains the hadron $H$ carrying the fractional light-cone momentum $z$ with respect to the parent parton $i$.

Fragmentation functions are nonperturbative yet universal objects, which provide essential information about the

\footnotetext{
a e-mail: f.feng@outlook.com

b e-mail: jiay@ihep.ac.cn

c e-mail: wlsang@swu.edu.cn (corresponding author)
}

hadronization mechanism. Similar to parton distribution functions, the scale dependence of FFs is governed by the celebrated Dokshitzer-Gribov-Lipatov-Altarelli-Parisi (DGLAP) equation:

$$
\begin{aligned}
& \frac{d}{d \ln \mu^{2}} D_{i \rightarrow H}(z, \mu) \\
& =\sum_{i} \int_{z}^{1} \frac{d y}{y} P_{j i}\left(y, \alpha_{s}(\mu)\right) D_{j \rightarrow H}\left(\frac{z}{y}, \mu\right)
\end{aligned}
$$

with $P_{i j}(y)$ the corresponding splitting kernel. The $\mu$ dependence of the fragmentation function conspires to compensate the $\mu$ dependence of $d \hat{\sigma}$ in (1), so that the physical cross section no longer depends on this artificial scale.

Fragmentation functions for quark and gluon into light hadrons such as $\pi, \rho$, proton, etc. are genuinely nonperturbative objects, which can only be extracted from experiments. There has emerged intensive interest in recent years toward explaining the LHC data for heavy quarkonium such as $J / \psi$, $\eta_{c}, \chi_{c J}$ production at large $p_{\perp}$, thus a thorough understanding of quarkonium FFs become important. Fortunately, because the heavy quark mass $m_{Q}$ is much greater than the intrinsic QCD scale $\Lambda_{\mathrm{QCD}}$, the FFs for a heavy quarkonium need not be the entirely non-perturbative object. In fact, owing to the weak QCD coupling at the length scale $\sim 1 / m_{Q}$, together with the nonrelativistic nature of heavy quarkonium, the factorization approach based on the nonrelativistic QCD (NRQCD) [2] can be invoked to further factorize the quarkonium FFs as the sum of products of perturbatively-calculable short-distance coefficients (SDCs) and long-distance yet universal NRQCD matrix elements $[3,4]$. To be specific, let us take the charm quark fragmentation into a charmonium $H$ as a concrete example. The NRQCD factorization theorem indicates that 


$$
\begin{aligned}
& D_{c \rightarrow H}(z, \mu)=\frac{d_{1}(z, \mu)}{m^{3}}\left\langle 0\left|\mathcal{O}_{1}^{H}\left({ }^{1} S_{0}\right)\right| 0\right\rangle \\
&+\frac{d_{8}(z, \mu)}{m^{3}}\left\langle 0\left|\mathcal{O}_{8}^{H}\left({ }^{1} S_{0}\right)\right| 0\right\rangle+\cdots .
\end{aligned}
$$

For the purpose of this work, we are only interested in the NRQCD production operators with the quantum number ${ }^{1} S_{0}$ :

$$
\begin{aligned}
& \mathcal{O}_{1}^{H}\left({ }^{1} S_{0}\right)=\chi^{\dagger} \psi \sum_{X}|H+X\rangle\langle H+X| \psi^{\dagger} \chi, \\
& \mathcal{O}_{8}^{H}\left({ }^{1} S_{0}\right)=\chi^{\dagger} T^{a} \psi \sum_{X}|H+X\rangle\langle H+X| \psi^{\dagger} T^{a} \chi,
\end{aligned}
$$

where $\psi\left(\chi^{\dagger}\right)$ annihilates a heavy (anti-)quark, respectively. $T^{a}\left(a=1, \ldots, N_{c}^{2}-1\right)$ represents the generators of $S U\left(N_{c}\right)$ group in the fundamental representation. $d_{1}(z, \mu)$ and $d_{8}(z, \mu)$ in (3) signify the SDCs affiliated with the respective production channels.

During the past two decades, the SDCs associated with various quarkonium fragmentation functions have been computed in NRQCD factorization framework. The heavy quark fragmentation into $S$-wave charmonia was computed at the lowest order both in $\alpha_{s}$ and velocity long ago [3,5-9]. The relativistic corrections to the heavy quark fragmenting into the $S$-wave charmonia were addressed in Refs. [10-12]. The perturbative corrections for heavy quark fragmentation into $S$ wave charmonia were evaluated in Refs. [13-15]. The heavy quark fragmenting into the $P$-wave quarkonia was investigated in Refs. [16-20]. On the other hand, the fragmentation functions for quarkonium with different flavor $\left(B_{c} / B_{c}^{*}\right)$ from charm/bottom quark [21-23] have also been calculated. Very recently, the authors of [24] computed the fragmentation functions for the spin-singlet quarkonium by a quark, which has a distinct flavor with the constituent quark in the quarkonium.

The gluon fragmentation into the $S$-wave quarkonium was originally calculated in Refs. $[4,25,26]$. The analytic expression for the gluon fragmenting into a vector quarkonium at LO in $\alpha_{s}$ was presented in Ref. [27]. The relativistic corrections to fragmentation functions for the $S$-wave quarkonium were computed in Refs. [27-30]. The NLO perturbative corrections for gluon fragmenting into $S$-wave quarkonia were studied in Refs. [31-36]. The gluon-to- $P$-wave quarkonia fragmentation functions were evaluated in [37-41].

The NLO perturbative corrections to $g \rightarrow{ }^{1} S_{0}^{(1,8)}$ channels of quarkonium have recently been considered in [34-36]. This provides valuable information for a thorough understanding of $\eta_{c}, h_{c}$ and $J / \psi$ production and polarization at large $p_{\perp}$. To facilitate a more realistic phenomenological analysis, one should also consider the analogous NLO correction for quark fragmentation. It is the aim of this work to compute the NLO perturbative corrections for the heavy quark to ${ }^{1} S_{0}^{(1,8)}$ channel of charmonia (bottomonia).
Our starting point is the gauge-invariant definition for the quark fragmentation functions pioneered by Collins and Soper long ago [42]. Note that this operator definition was first used by Ma to compute the quarkonium FFs in NRQCD factorization [6]. According to the operator definition [42] (see also [9]), the desired $c$-to- $H$ fragmentation function in QCD reads

$$
\begin{aligned}
& D_{c \rightarrow H}(z, \mu)=\frac{z^{D-3}}{2 \pi \times 4 \times N_{c}} \int_{-\infty}^{+\infty} d x^{-} e^{-i k^{+} x^{-}} \\
& \times \operatorname{tr}\left[h \left\langle0\left|\Psi(0) \Phi^{\dagger}\left(0,0, \mathbf{0}_{\perp}\right) \sum_{X}\right| H(P)\right.\right. \\
& \left.+X\rangle\left\langle H(P)+X\left|\Phi\left(0, x^{-}, \mathbf{0}_{\perp}\right) \bar{\Psi}\left(0, x^{-}, \mathbf{0}_{\perp}\right)\right| 0\right\rangle\right] .
\end{aligned}
$$

The light-cone coordinate $V^{\mu}=\left(V^{+}, V^{-}, \mathbf{V}_{\perp}\right)$ has been used, and $n^{\mu}=\left(0,1, \mathbf{0}_{\perp}\right)$ is a null reference 4 -vector. The variable $z$ denotes the fraction of the + -momentum carried by $H$ with respect to the charm quark, $k^{+}=P^{+} / z$ is the + -component momentum injected by the charm quark field operator $\Psi(x) . D=4-2 \varepsilon$ signifies the space-time dimensions. $\mu$ is the renormalization scale for this composite operator. The insertion of the intermediate states implies that in the asymptotic future, one only needs project out those outstates that contain a charmonium $H$ carrying definite momentum $P^{\mu}$ plus any unobserved hadrons, which are collectively denoted by the symbol $X$.

The eikonal factor $\Phi\left(0, x^{-}, \mathbf{0}_{\perp}\right)$ in (5) is the path-ordered exponential of the gluon field, whose role is to ensure the gauge invariance of the FF:

$\Phi\left(0, x^{-}, \mathbf{0}_{\perp}\right)=\mathrm{P} \exp \left[i g_{s} \int_{x^{-}}^{\infty} d y^{-} n \cdot A\left(0^{+}, y^{-}, \mathbf{0}_{\perp}\right)\right]$,

where P implies the path-ordering, $g_{s}$ is the QCD coupling constant, and $A^{\mu}$ denotes the matrix-valued gluon field in $S U\left(N_{c}\right)$ fundamental representation.

We can appeal to standard perturbative matching method to determine the SDCs $d_{1,8}(z, \mu)$ in (3), by replacing the physical quarkonium $H$ by a fictitious one $c \bar{c}\left({ }^{1} S_{0}^{(1,8)}\right)$. For the QCD part of the amplitude, one needs to project the $c \bar{c}$ pair onto the intended spin/orbital/color states. It is convenient to employ the covariant projector to expedite the calculation [43]:

$\Pi_{1,8}=\frac{1}{\sqrt{8 m^{3}}}\left(\frac{P}{2}-m\right) \gamma_{5}\left(\frac{P}{2}+m\right) \otimes \mathcal{C}_{1,8}$,

with $P^{\mu}$ designating the total momentum of the $c \bar{c}$ pair, and the color projectors $\mathcal{C}_{1,8}$ given by

$\mathcal{C}_{1}=\frac{1_{c}}{\sqrt{N_{c}}}$

$\mathcal{C}_{8}^{a}=\sqrt{2} T^{a}$ 
Fig. 1 Some representative Feynman diagrams for the charm quark fragmentation function $D_{c \rightarrow c \bar{c}\left({ }^{1} S_{0}^{(1,8)}\right)}(z)$ at LO and NLO in $\alpha_{s}$. The cap represents the injected quark field operator, while the double line signifies the eikonal line

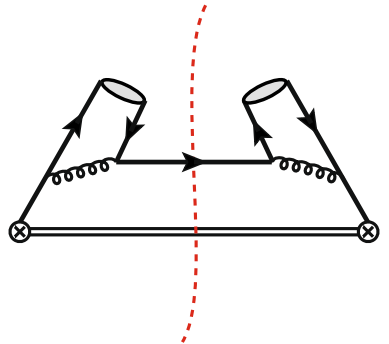

a $\mathrm{LO}$ $1_{c}$ is the $N_{c}$-dimensional unit matrix. Because we are only interested in the LO accuracy in velocity expansion, we have neglected the relative momentum between $c$ and $\bar{c}$ in (7), consequently we approximate $P^{2}$ by $4 m^{2}$.

Although (5) is manifestly gauge-invariant, it is simplest to specialize to the Feynman gauge in practical calculation. Dimensional regularization is used throughout this work to regularize both UV and IR divergences. We use two private packages to automatically generate the Feynman diagrams and the associated cut amplitudes that correspond to the perturbative fragmentation function defined in (5). One is based on the package Qgraf [44], and the other based on FeynArts [45]. We have implemented Feynman rules involving the eikonal propagator and vertex [42] as well as those for conventional QCD propagators and vertices. Some representative Feynman diagrams for perturbative FF through NLO in $\alpha_{s}$ are shown in Fig. 1.

With the aid of the covariant projector (7), after obtaining the expressions for the cut amplitudes, we then utilize the packages FeynCalc/FormLink [46,47] to conduct the Dirac/color trace algebra. We also use the package Apart [48] to simplify the amplitude by the method of partial fraction, to make the integrand in loop integrals simpler.

A peculiar structure of the fragmentation function originates from its cut topology, with insertion of the asymptotic out-state in (5). The corresponding phase-space integration measure through the cut becomes [28,29]

$$
\begin{aligned}
d \Phi_{n}= & \frac{8 \pi m}{S_{n}} \delta\left(k^{+}-P^{+}-\sum_{i=1}^{n} k_{i}^{+}\right) \\
& \times \prod_{i=1}^{n} \frac{d k_{i}^{+}}{2 k_{i}^{+}} \frac{d^{D-2} k_{i \perp}}{(2 \pi)^{D-1}} \theta\left(k_{i}^{+}\right)
\end{aligned}
$$

where $k_{i}(i=1,2)$ stands for the momentum of the $i$-th on-shell parton that passes through the cut, and $S_{n}$ is the statistical factor for $n$ identical partons. For our purpose, suffices it to set $S_{n}=1$. It is important to note that integration over $k_{i}^{+}$can be transformed into a parametric integration in a finite interval, but the integration over the transverse momentum $k_{i, \perp}$ are utterly unbounded, i.e., from $-\infty$ to $+\infty$. This feature indicates that the integration over $k_{i, \perp}$ could be regarded as loop integration in $D-2$-dimensional spacetime.

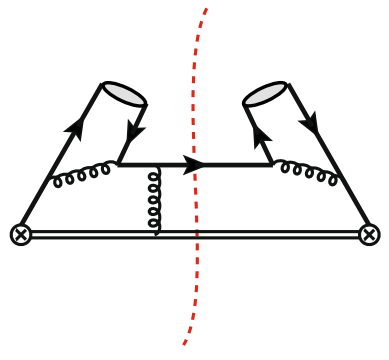

b NLO (virtual)

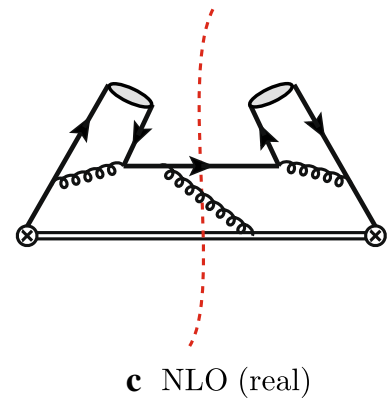

c $\mathrm{NLO}($ real $)$
As first noticed in $[35,36]$, some technical nuisance may arise in utilizing the standard integration-by-part (IBP) technique to tackle the NLO real corrections, whereas it is quite safe to apply IBP to handle NLO virtual correction. As expounded in [35], in this work we decide to utilize the sector decomposition technique $[49,50]$ to evaluate all the NLO real correction diagrams,

Through perturbative matching procedure, we are able to deduce the SDCs appearing in (3) order by order in $\alpha_{s}$ :

$d_{1,8}(z, \mu)=d_{1,8}^{\mathrm{LO}}(z)+\frac{\alpha_{s}(\mu)}{\pi} d_{1,8}^{\mathrm{NLO}}(z, \mu)+\cdots$.

The desired LO SDCs in $D=4-2 \epsilon$ dimensions turn out to be

$$
\begin{aligned}
& d_{1}^{\mathrm{LO}}(z)=\frac{16 \alpha_{s}^{2}(1-z)^{2} z(4 \pi)^{\epsilon}(2-z)^{-2 \epsilon} \Gamma(1+\epsilon)}{243(2-z)^{6}}, \\
& \times\left[\left(48+8 z^{2}-8 z^{3}+3 z^{4}\right)\right. \\
& \left.-96(1-z) \epsilon+\left(48-96 z+40 z^{2}+8 z^{3}-3 z^{4}\right) \epsilon^{2}\right], \\
& d_{8}^{\mathrm{LO}}(z)=\frac{\alpha_{s}^{2}(1-z)^{2} z(4 \pi)^{\epsilon}(2-z)^{-2 \epsilon} \Gamma(1+\epsilon)}{162(2-z)^{6}} \\
& \quad \times\left[\left(48+8 z^{2}-8 z^{3}+3 z^{4}\right)\right. \\
& \left.-96(1-z) \epsilon+\left(48-96 z+40 z^{2}+8 z^{3}-3 z^{4}\right) \epsilon^{2}\right] .
\end{aligned}
$$

Setting $\epsilon=0$, our result for $d_{1}^{\mathrm{LO}}$ is compatible with [3], and our result for $d_{8}^{\mathrm{LO}}$ agrees with [17].

Summing both real and virtual NLO corrections, implementing standard renormalization procedure for QCD lagrangian (the QCD coupling constant is renormalized under the $\overline{\mathrm{MS}}$ scheme), we find that the infrared pole indeed disappear in the NLO SDCs in both color-singlet and octet channels. However, there still scurvies an z-dependent single UV pole. This is simply the symptom that the fragmentation function at NLO still requires an additional operator renormalization $[9,42]$ :

$$
\begin{aligned}
& D_{c \rightarrow H}^{\overline{\mathrm{MS}}}(z, \mu)=D_{c \rightarrow H}^{\text {bare }}(z, \mu) \\
& -\frac{1}{\epsilon} \frac{\alpha_{s}}{2 \pi} \int_{z}^{1} \frac{d y}{y} P_{c c}(y) D_{c \rightarrow H}(z / y, \mu) \\
& -\frac{1}{\epsilon} \frac{\alpha_{s}}{2 \pi} \int_{z}^{1} \frac{d y}{y} P_{g c}(y) D_{g \rightarrow H}(z / y, \mu),
\end{aligned}
$$


Table 1 Numerical values of non-logarithmic color-singlet coefficient functions $c_{1,2,3}^{(1)}(z)$ as introduced in (17). We caution that the actual values of $c_{i}^{(1)}(z)$ should be multiplied by an extra factor $10^{-2}$

\begin{tabular}{lllllrrr}
\hline$z$ & $c_{1}^{(1)}(z)$ & $c_{2}^{(1)}(z)$ & $c_{3}^{(1)}(z)$ & $z$ & $c_{1}^{(1)}(z)$ & $c_{2}^{(1)}(z)$ & $c_{3}^{(1)}(z)$ \\
\hline 0.05 & $-84.7925(6)$ & 0.26142 & 0.25443 & 0.55 & $-18.8763(3)$ & 0.91199 \\
0.10 & $-54.2569(8)$ & 0.42309 & 0.39228 & 0.60 & $-13.5185(3)$ & 0.89995 & -0.45891 \\
0.15 & $-45.6338(2)$ & 0.54973 & 0.47410 & 0.65 & $-7.8484(3)$ & 0.88283 & -0.77734 \\
0.20 & $-41.7388(6)$ & 0.65275 & 0.50740 & 0.70 & $-2.1861(4)$ & 0.86303 & -0.79912 \\
0.25 & $-39.2176(5)$ & 0.73655 & 0.49315 & 0.75 & $3.0117(3)$ & 0.83987 & -0.68278 \\
0.30 & $-36.9564(2)$ & 0.80310 & 0.43092 & 0.80 & $7.1367(2)$ & 0.80516 & -0.42365 \\
0.35 & $-34.4680(2)$ & 0.85342 & 0.32112 & 0.85 & $9.4336(3)$ & 0.73635 \\
0.40 & $-31.5042(2)$ & 0.88828 & 0.16675 & 0.90 & $9.0611(2)$ & 0.58954 & -0.76013 \\
0.45 & $-27.9368(2)$ & 0.90861 & -0.02492 & 0.95 & $5.3646(3)$ & 0.31034 & 2.16314 \\
0.50 & $-23.7186(3)$ & 0.91581 & -0.24051 & 0.99 & $0.6854(2)$ & 0.03175 & 0.23972 \\
\hline
\end{tabular}

Table 2 Numerical values of non-logarithmic color-octet coefficient functions $c_{1,2,3}^{(1)}(z)$ as introduced in (17). We caution that the actual values of $c_{1}^{(1)}(z), c_{2}^{(1)}(z)$ and $c_{3}^{(1)}(z)$ should be multiplied by a factor $10^{-2}$

\begin{tabular}{rrrrrrrr}
\hline$z$ & $c_{1}^{(1)}(z)$ & $c_{2}^{(1)}(z)$ & $c_{3}^{(1)}(z)$ & $z$ & $c_{1}^{(1)}(z)$ & $c_{2}^{(1)}(z)$ & $c_{3}^{(1)}(z)$ \\
\hline 0.05 & $-89.6874(4)$ & 0.02451 & 0.02385 & 0.55 & $10.0661(5)$ & 0.08550 & -0.04302 \\
0.10 & $-26.5678(3)$ & 0.03967 & 0.03678 & 0.60 & $9.8386(4)$ & 0.08437 & -0.06097 \\
0.15 & $-7.4488(4)$ & 0.05154 & 0.04445 & 0.65 & $9.4633(7)$ & 0.08276 & -0.07288 \\
0.20 & $0.9549(3)$ & 0.06120 & 0.04757 & 0.70 & $7.8382(6)$ & 0.08091 & -0.07492 \\
0.25 & $5.2463(2)$ & 0.06905 & 0.04623 & 0.75 & $5.3851(4)$ & 0.07874 & -0.06401 \\
0.30 & $7.5974(3)$ & 0.07529 & 0.04040 & 0.80 & $2.7520(4)$ & 0.07548 & -0.03972 \\
0.35 & $8.9190(4)$ & 0.08001 & 0.03011 & 0.85 & $0.2366(5)$ & 0.06903 & -0.00713 \\
0.40 & $9.6491(3)$ & 0.08328 & 0.01563 & 0.90 & $-1.5717(4)$ & 0.05527 & 0.02028 \\
0.45 & $10.0142(5)$ & 0.08518 & -0.00234 & 0.95 & $-1.7326(5)$ & 0.02909 \\
0.50 & $10.1334(3)$ & 0.08586 & -0.02255 & 0.99 & $-0.2953(0)$ & 0.00298 & 0.02247 \\
\hline
\end{tabular}

where $P_{c c}(y)$ and $P_{g c}(y)$ represent the Altarelli-Parisi splitting kernels for $c \rightarrow c$ and $c \rightarrow g$ respectively:

$$
\begin{aligned}
& P_{c c}(y)=C_{F}\left[\frac{1+y^{2}}{(1-y)_{+}}+\frac{3}{2} \delta(1-y)\right], \\
& P_{g c}(y)=C_{F} \frac{1+(1-y)^{2}}{y} .
\end{aligned}
$$

Note in (12) the UV pole is subtracted in accordance with the $\overline{\mathrm{MS}}$ scheme.

Finally, the renormalized SDCs at NLO in $\alpha_{s}$ can be parameterized in the following form:

$$
\begin{aligned}
d_{1,8}^{\mathrm{NLO}}(z, \mu)= & \beta_{0} \ln \frac{\mu_{R}^{2}}{m^{2}} d_{1,8}^{\mathrm{LO}}(z) \\
& +\frac{1}{2} \ln \frac{\mu^{2}}{m^{2}}\left[\int_{z}^{1} \frac{d y}{y} P_{c c}(y) d_{1,8}^{\mathrm{LO}}(z / y)\right. \\
& \left.+\int_{z}^{1} \frac{d y}{y} P_{g c}(y) d_{g \rightarrow H}^{\mathrm{LO}}(z / y)\right]+f_{1,8}(z),
\end{aligned}
$$

with $\beta_{0}$ the one-loop QCD $\beta$ function.

With the aid of $D$-dimensional expressions for the LO SDCs in (11), we can deduce the coefficients of the $\ln \mu^{2}$ terms analytically. For the color-singlet channel, we have

$$
\begin{aligned}
& \int_{z}^{1} \frac{d y}{y} P_{c c}(y) d_{1}^{\mathrm{LO}}(z / y)=\frac{16 \alpha_{s}^{2}}{10935(2-z)^{6}} \\
& \quad \times\left[120 z\left(48+8 z^{2}-8 z^{3}+3 z^{4}\right)(1-z)^{2} \ln (1-z)\right. \\
& \quad-15\left(5632-16320 z+19008 z^{2}-10928 z^{3}\right. \\
& \left.\quad+3072 z^{4}-372 z^{5}+20 z^{6}-9 z^{7}\right) \ln (2-z) \\
& \quad-15 z\left(192-192 z-272 z^{2}+288 z^{3}\right. \\
& \left.\quad+36 z^{4}-76 z^{5}+21 z^{6}\right) \ln z \\
& \quad+2\left(30848-78352 z+76400 z^{2}-37160 z^{3}\right. \\
& \left.\left.\quad+10420 z^{4}-1553 z^{5}+192 z^{6}\right)(1-z)\right] \\
& \int_{z}^{1} \frac{d y}{y} P_{g c}(y) d_{g \rightarrow{ }^{1}}^{\mathrm{LO}} S_{0}^{[1]}(z / y)=\frac{\alpha_{s}^{2}}{81 z} \\
& \quad \times\left[-6(z+2) z \operatorname{Li}_{2}(z)-3 z^{2} \ln z+6(1-z)(2 z+1) \ln (1-z)\right.
\end{aligned}
$$



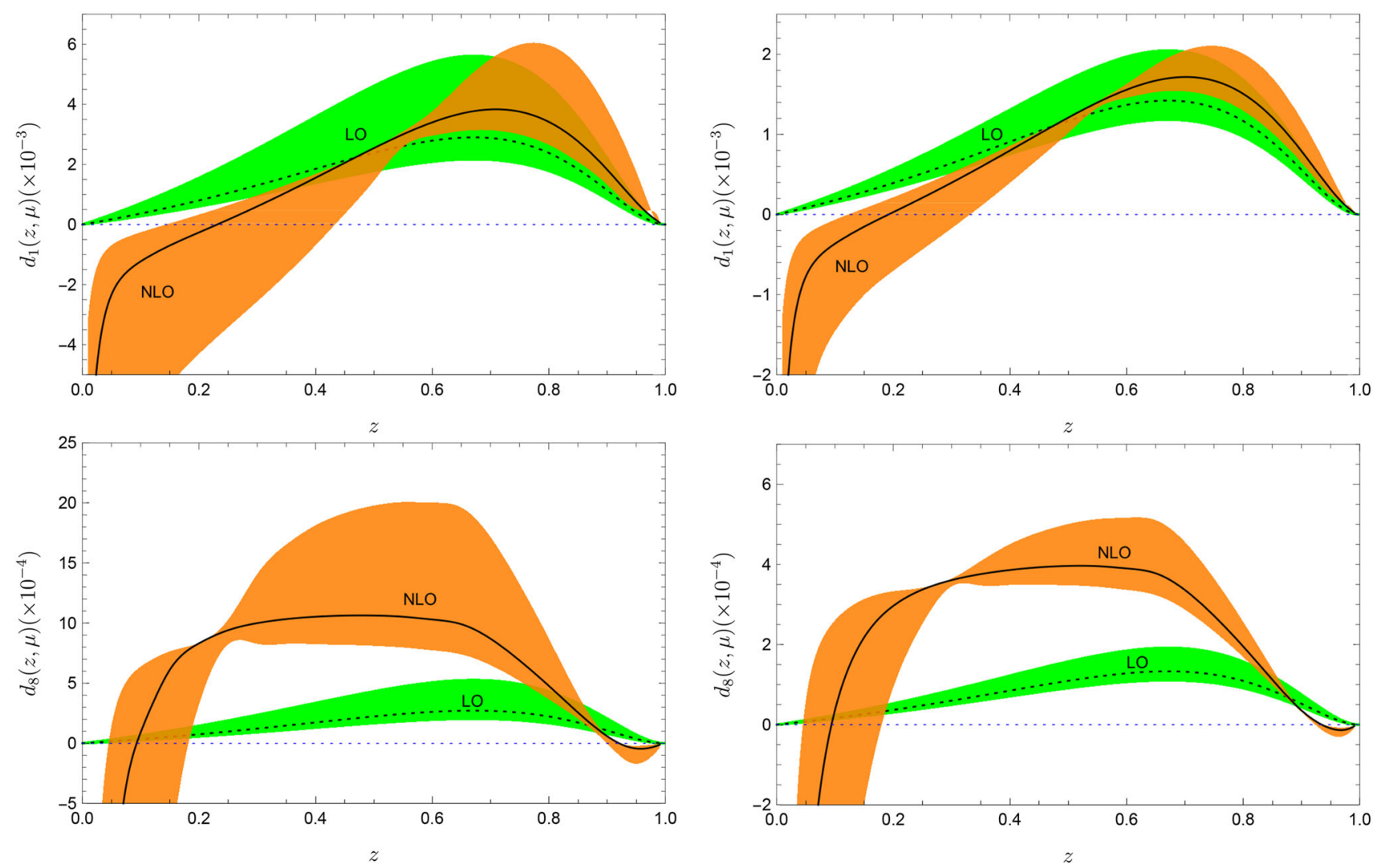

Fig. 2 The SDCs $d_{1,8}(z)$ associated with heavy quark fragmentation functions, accurate up to NLO in $\alpha_{s}$. The two figures in the left column correspond to $d_{1,8}(z)$ for charm quark fragmentation into charmonium, while the two figures in the right column correspond to those for bottom

$$
\left.\times 2+\left(2 \pi^{2}-18\right) z+\left(\pi^{2}+12\right) z^{2}+4 z^{3}\right]
$$

For the color-octet channel, the coefficients of $\ln \mu^{2}$ term read

$$
\begin{aligned}
& \int_{z}^{1} \frac{d y}{y} P_{c c}(y) d_{8}^{\mathrm{LO}}(z / y)=\frac{\alpha_{s}^{2}}{7290(2-z)^{6}} \\
& \quad \times\left[120 z\left(48+8 z^{2}-8 z^{3}+3 z^{4}\right)(1-z)^{2} \ln (1-z)\right. \\
& \quad-15\left(5632-16320 z+19008 z^{2}-10928 z^{3}\right. \\
& \left.\quad+3072 z^{4}-372 z^{5}+20 z^{6}-9 z^{7}\right) \ln (2-z) \\
& \quad-15 z\left(192-192 z-272 z^{2}+288 z^{3}+36 z^{4}-76 z^{5}+21 z^{6}\right) \ln z \\
& +61696-218400 z+309504 z^{2}-227120 z^{3}+95160 z^{4} \\
& \left.\quad-23946 z^{5}+3490 z^{6}-384 z^{7}\right], \\
& \int_{z}^{1} \frac{d y}{y} P_{g c}(y) d_{g \rightarrow{ }^{1}}{ }^{\mathrm{LO}} S_{0}^{[8]}(z / y)=\frac{10 \alpha_{s}^{2}}{432 z} \\
& \quad \times\left[-6(z+2) z \operatorname{Li}_{2}(z)-3 z^{2} \ln z+6\left(-2 z^{2}+z+1\right) \ln (1-z)\right. \\
& \left.\quad+2+2\left(\pi^{2}-9\right) z+\left(12+\pi^{2}\right) z^{2}+4 z^{3}\right] .
\end{aligned}
$$

quark fragmentation. The uncertainty bands are derived by varying the factorization scale $\mu$ from $m_{Q}$ to $3 m_{Q}$, with the central value taken at $2 m_{Q}$

We notice the occurrence of $1 / z$ singularity in the integrals involving $P_{g c}$.

The non-logarithmic terms can be decomposed in terms of distinct flavor structure:

$f_{1,8}(z)=\alpha_{s}^{2}\left[c_{1}^{(1,8)}(z)+n_{L} c_{2}^{1,8}(z)+n_{H} c_{3}^{(1,8)}(z)\right]$

where $n_{L}$ denotes the number of light quarks, and $n_{H}=$ 1 is the number of heavy quark. The numerical values of individual coefficient functions $c_{i}^{(1,8)}(z)(i=1,2,3)$ have been tabulated in Tables 1 and 2 .

For a concrete investigation of the heavy quark fragmentation functions, we take the following values for the one-loop pole mass of charm and bottom quarks:

$m_{c}=1.4 \mathrm{GeV}, m_{b}=4.6 \mathrm{GeV}$.

The running QCD coupling is computed with two-loop accuracy with the aid of the package RunDec [51]. We have taken $n_{L}=3,4$ for charmonium and bottomonium, respectively, so that $n_{f}=n_{L}+1$.

The profiles of the SDCs $d_{1,8}(z)$ through the NLO in $\alpha_{s}$ are displayed in Fig. 2 for $c$ to charmonium and $b$ to bot- 
tomonium, respectively. We have equated the renormalization scale $\mu_{R}$ and factorization scale $\mu$ in (14) for simplicity. The central curves are obtained by setting $\mu=2 m_{Q}$, while the uncertainty bands are obtained by varying $\mu$ from $m_{Q}$ to $3 m_{Q}$. Aa can be clearly visualized, the NLO perturbative corrections have a significant impact on both color-singlet and octet channels, for both charm and bottom fragmentation function, including which would qualitatively modify the shapes of LO fragmentation functions. Therefore, it appears mandatory to include the NLO QCD corrections in the future phenomenological analysis.

Note added After this work was completed and while we were preparing the manuscript, very recently a preprint [52] has appeared, which also computes the NLO perturbative corrections to the heavy quark fragmentation into a ${ }^{1} S_{0}^{(1)}$ quarkonium. Their numerical results appear to be compatible with ours in this color-singlet channel.

Acknowledgements The work of F. F. is supported by the National Natural Science Foundation of China under Grant no. 11875318, no. 11505285 , and by the Yue Qi Young Scholar Project in CUMTB. The work of Y. J. is supported in part by the National Natural Science Foundation of China under Grants no. 11925506, 11875263, no. 12070131001 (CRC110 by DFG and NSFC). The work of W.-L. S. is supported by the National Natural Science Foundation of China under Grants no. 11975187 and the Natural Science Foundation of ChongQing under Grant no. cstc2019jcyj-msxmX0479.

Data Availability Statement This manuscript has no associated data or the data will not be deposited. [Authors' comment: All the figure and the numerical predictions can be obtained from the formulas presented in the paper, so the data do not need to be deposited.]

Open Access This article is licensed under a Creative Commons Attribution 4.0 International License, which permits use, sharing, adaptation, distribution and reproduction in any medium or format, as long as you give appropriate credit to the original author(s) and the source, provide a link to the Creative Commons licence, and indicate if changes were made. The images or other third party material in this article are included in the article's Creative Commons licence, unless indicated otherwise in a credit line to the material. If material is not included in the article's Creative Commons licence and your intended use is not permitted by statutory regulation or exceeds the permitted use, you will need to obtain permission directly from the copyright holder. To view a copy of this licence, visit http://creativecomm ons.org/licenses/by/4.0/.

Funded by SCOAP ${ }^{3}$.

\section{References}

1. J.C. Collins, D.E. Soper, G.F. Sterman, Adv. Ser. Direct. High Energy Phys. 5, 1-91 (1989). https://doi.org/10.1142/ 9789814503266_0001. arXiv:hep-ph/0409313

2. G.T. Bodwin, E. Braaten, G.P. Lepage, Phys. Rev. D 51, 1125-1171 (1995). https://doi.org/10.1103/PhysRevD.55.5853. arXiv:hep-ph/9407339 [Erratum: Phys. Rev. D 55, 5853 (1997)]

3. E. Braaten, K.M. Cheung, T.C. Yuan, Phys. Rev. D 48, 4230-4235 (1993). https://doi.org/10.1103/PhysRevD.48.4230. arXiv:hep-ph/9302307
4. E. Braaten, T.C. Yuan, Phys. Rev. Lett. 71, 16731676 (1993). https://doi.org/10.1103/PhysRevLett.71.1673. arXiv:hep-ph/9303205

5. A.F. Falk, M.E. Luke, M.J. Savage, M.B. Wise, Phys. Lett. B 312, 486-490 (1993). https://doi.org/10.1016/0370-2693(93)90986-R. arXiv:hep-ph/9305260

6. J.P. Ma, Phys. Lett. B 332, 398-404 (1994). https://doi.org/10. 1016/0370-2693(94)91271-8. arXiv:hep-ph/9401249

7. Y.Q. Ma, J.W. Qiu, H. Zhang, Phys. Rev. D 89(9), 094029 (2014). https://doi.org/10.1103/PhysRevD.89.094029. arXiv:1311.7078 [hep-ph]

8. H.Zhang, QCD factorization for heavy quarkonium production and fragmentation functions. A Dissertation for the Degree of Doctor of Philosophy in Physics, Stony Brook University (2014)

9. G.T. Bodwin, H.S. Chung, U.R. Kim, J. Lee, Phys. Rev. D 91(7), 074013 (2015). https://doi.org/10.1103/PhysRevD.91. 074013. arXiv:1412.7106 [hep-ph]

10. A.P. Martynenko, Phys. Rev. D 72, 074022 (2005). https://doi.org/ 10.1103/PhysRevD.72.074022. arXiv:hep-ph/0506324

11. W. Sang, L. Yang, Y. Chen, Phys. Rev. D 80, 014013 (2009). https:// doi.org/10.1103/PhysRevD.80.014013

12. D. Yang, W. Zhang, Chin. Phys. C 43(8), 083101 (2019). https:// doi.org/10.1088/1674-1137/43/8/083101. arXiv:1905.02923 [hep-ph]

13. R. Sepahvand, S. Dadfar, Phys. Rev. D 95(3), 034012 (2017). https://doi.org/10.1103/PhysRevD.95.034012

14. X.C. Zheng, C.H. Chang, T.F. Feng, X.G. Wu, Phys. Rev. D 100(3), 034004 (2019). https://doi.org/10.1103/PhysRevD.100. 034004. arXiv:1901.03477 [hep-ph]

15. X.C. Zheng, C.H. Chang, X.G. Wu, Phys. Rev. D 100(1), 014005 (2019). https://doi.org/10.1103/PhysRevD.100.014005. arXiv:1905.09171 [hep-ph]

16. Y.Q. Chen, Phys. Rev. D 48, 5181-5189 (1993). https://doi.org/10. 1103/PhysRevD.48.5181

17. T.C. Yuan, Phys. Rev. D 50, 5664-5675 (1994). https://doi.org/10. 1103/PhysRevD.50.5664. arXiv:hep-ph/9405348

18. J.P. Ma, Phys. Rev. D 53, 1185-1190 (1996). https://doi.org/10. 1103/PhysRevD.53.1185. arXiv:hep-ph/9504263

19. Y. Jia, W.L. Sang, J. Xu, Phys. Rev. D 86, 074023 (2012). https:// doi.org/10.1103/PhysRevD.86.074023. arXiv:1206.5785 [hep-ph]

20. Y.Q. Ma, J.W. Qiu, H. Zhang, Phys. Rev. D 89(9), 094030 (2014). https://doi.org/10.1103/PhysRevD.89.094030. arXiv:1401.0524 [hep-ph]

21. C.R. Ji, F. Amiri, Phys. Rev. D 35, 3318 (1987). https://doi.org/10. 1103/PhysRevD.35.3318

22. C.H. Chang, Y.Q. Chen, Phys. Rev. D 46, 3845 (1992). https://doi. org/10.1103/PhysRevD.46.3845 [Erratum: Phys. Rev. D 50, 6013 (1994)]

23. E. Braaten, K. Cheung, T.C. Yuan, Phys. Rev. D 48(11), R5049 (1993). https://doi.org/10.1103/PhysRevD.48.R5049. arXiv:hep-ph/9305206

24. X.C. Zheng, Z.Y. Zhang, X.G. Wu, Phys. Rev. D 103(7), 074004 (2021). https://doi.org/10.1103/PhysRevD.103.074004. arXiv:2101.01527 [hep-ph]

25. E. Braaten, T.C. Yuan, Phys. Rev. D 52, 6627-6629 (1995). https:// doi.org/10.1103/PhysRevD.52.6627. arXiv:hep-ph/9507398

26. P.L. Cho, M.B. Wise, S.P. Trivedi, Phys. Rev. D 51, R2039R2043 (1995). https://doi.org/10.1103/PhysRevD.51.R2039. arXiv:hep-ph/9408352

27. P. Zhang, Y.Q. Ma, Q. Chen, K.T. Chao, Phys. Rev. D 96(9), 094016 (2017). https://doi.org/10.1103/PhysRevD.96. 094016. arXiv:1708.01129 [hep-ph]

28. G.T. Bodwin, J. Lee, Phys. Rev. D 69, 054003 (2004). https://doi. org/10.1103/PhysRevD.69.054003. arXiv:hep-ph/0308016

29. G.T. Bodwin, U.R. Kim, J. Lee, JHEP 11, 020 (2012). https://doi. org/10.1007/JHEP11(2012)020. arXiv:1208.5301 [hep-ph] 
30. X. Gao, Y. Jia, L. Li, X. Xiong, Chin. Phys. C 41(2), 023103 (2017). https://doi.org/10.1088/1674-1137/41/2/023103. arXiv:1606.07455 [hep-ph]

31. M. Beneke, I.Z. Rothstein, Phys. Lett. B 372, 157-164 (1996). https://doi.org/10.1016/0370-2693(96)00030-5. arXiv:hep-ph/9509375 [Erratum: Phys. Lett. B 389, 769 (1996)]

32. E. Braaten, J. Lee, Nucl. Phys. B 586, 427-439 (2000). https://doi. org/10.1016/S0550-3213(00)00396-5. arXiv:hep-ph/0004228

33. P. Artoisenet, E. Braaten, JHEP 04, 121 (2015). https://doi.org/10. 1007/JHEP04(2015)121. arXiv:1412.3834 [hep-ph]

34. P. Artoisenet, E. Braaten, JHEP 01, 227 (2019). https://doi.org/10. 1007/JHEP01(2019)227. arXiv:1810.02448 [hep-ph]

35. F. Feng, Y. Jia, arXiv:1810.04138 [hep-ph]

36. P. Zhang, C.Y. Wang, X. Liu, Y.Q. Ma, C. Meng, K.T. Chao, JHEP 04, 116 (2019). https://doi.org/10.1007/JHEP04(2019)116. arXiv: 1810.07656 [hep-ph]

37. E. Braaten, T.C. Yuan, Phys. Rev. D 50, 3176-3180 (1994). https:// doi.org/10.1103/PhysRevD.50.3176. arXiv:hep-ph/9403401

38. J.P. Ma, Nucl. Phys. B 447, 405-424 (1995). https://doi.org/10. 1016/0550-3213(95)00270-3. arXiv:hep-ph/9503346

39. G. Hao, Y. Zuo, C.F. Qiao, arXiv:0911.5539 [hep-ph]

40. F. Feng, S. Ishaq, Y. Jia, J.Y. Zhang, Phys. Rev. D 102(1), 014038 (2020). https://doi.org/10.1103/PhysRevD.102.014038. arXiv:1712.09986 [hep-ph]

41. P. Zhang, C. Meng, Y.Q. Ma, K.T. Chao, arXiv:2011.04905 [hep$\mathrm{ph}]$
42. J.C. Collins, D.E. Soper, Nucl. Phys. B 194, 445-492 (1982). https://doi.org/10.1016/0550-3213(82)90021-9

43. A. Petrelli, M. Cacciari, M. Greco, F. Maltoni, M.L. Mangano, Nucl. Phys. B 514, 245-309 (1998). https://doi.org/10.1016/ S0550-3213(97)00801-8. arXiv:hep-ph/9707223

44. P. Nogueira, J. Comput. Phys. 105, 279-289 (1993). https://doi. org/10.1006/jcph.1993.1074

45. T. Hahn, Comput. Phys. Commun. 140, 418-431 (2001). https:// doi.org/10.1016/S0010-4655(01)00290-9. arXiv:hep-ph/0012260

46. R. Mertig, M. Bohm, A. Denner, Comput. Phys. Commun. 64, 345-359 (1991). https://doi.org/10.1016/0010-4655(91)90130-D

47. F. Feng, R. Mertig, arXiv:1212.3522 [hep-ph]

48. F. Feng, Comput. Phys. Commun. 183, 2158-2164 (2012). https:// doi.org/10.1016/j.cpc.2012.03.025. arXiv:1204.2314 [hep-ph]

49. T. Binoth, G. Heinrich, Nucl. Phys. B 585, 741-759 (2000). https:// doi.org/10.1016/S0550-3213(00)00429-6. arXiv:hep-ph/0004013

50. T. Binoth, G. Heinrich, Nucl. Phys. B 680, 375-388 (2004). https:// doi.org/10.1016/j.nuclphysb.2003.12.023. arXiv:hep-ph/0305234

51. K.G. Chetyrkin, J.H. Kuhn, M. Steinhauser, Comput. Phys. Commun. 133, 43-65 (2000). https://doi.org/10.1016/ S0010-4655(00)00155-7. arXiv:hep-ph/0004189

52. X.C. Zheng, X.G. Wu, X.D. Huang, arXiv:2105.14580 [hep-ph] 